\title{
Pulmonary Paragonimiasis with Coincidental Malignant Mesothelioma
}

\author{
Mariko Yamazaki ${ }^{1,4}$, Akihiko Ohwada ${ }^{1,4}$, Atsuko Miyaji ${ }^{1,4}$, Hiroshi Yamazaki ${ }^{7}$, \\ Takeshi Nara ${ }^{5}$, Syu Hirai ${ }^{2}$, Hiroaki Fujii ${ }^{6}$, Toshimasa Uekusa ${ }^{4}$, \\ Masaru Suzuki ${ }^{3}$, Akihiko Iwase ${ }^{1,4}$ and Kazuhisa Takahashi ${ }^{4}$
}

\begin{abstract}
A 72-year-old man patient was referred to our institution for evaluation and treatment of right pleural effusion. Eosinophilic pleural effusion and peripheral eosinophilia were identified during the course of hospitalization. Pulmonary paragonimiasis was confirmed by the presence of paragonimus-specific IgG antibodies for Paragonimus (P.) westermani and P. miyazakii in his serum. Although Praziquantel, a highly effective agent for the treatment of lung flukes was repeatedly administered, the pleural effusion did not subside and the patient's condition gradually deteriorated until his death due to circulatory insufficiency. Postmortem examination revealed malignant mesothelioma of the sarcomatous type encasing the right lung and heart. Cardiac involvement accompanied with old and recent-onset myocardial ischemic changes resulted in death of this patient. Here, we report a very rare case of malignant mesothelioma with a concomitant infection of parasitic lung fluke.
\end{abstract}

Key words: paragonimiasis, malignant mesothelioma, eosinophilia

(Inter Med 47: 1027-1031, 2008)

(DOI: 10.2169/internalmedicine.47.0852)

\section{Introduction}

Paragonimiasis, a parasitic infection caused by lung flukes, Paragonimus (P.) westermani and P. miyazakii is rarely encountered in Japan but has been a representative parasitic infection of the lungs resulting from the consumption of improperly cooked or raw secondary intermediate hosts containing the parasite, such as fresh water crabs, crayfish and wild boar meat (1). Pulmonary paragonimiasis, cerebral paragonimiasis and subcutaneous paragonimiasis have been previously reported in the literature (2-4). We herein report an elderly Japanese man patient with encapsulated eosinophilic pleural effusion and peripheral eosinophilia diagnosed as paragonimiasis from the positive result of enzyme-linked immunosorbent assay (ELISA) for Paragonimus-specific IgG antibodies. The patient died of cardiac failure without any signs of improvement despite comprehensive and extensive treatment with Praziquantel, a potent anti-parasitic agent. Concomitant malignant mesothelioma was revealed during postmortem examination.

\section{Case Report}

A 72-year-old man patient was referred to our institution for evaluation and treatment for right-sided pleural effusion. The patient presented with chief complaints of persistent cough and progressive dyspnea which began approximately two months earlier, as well as right-sided pleural effusion. Thoracentesis performed at his previous hospital revealed

${ }^{1}$ Department of Respiratory Medicine, Juntendo Tokyo Koto Geriatric Medical Center, Tokyo, ${ }^{2}$ Department of Pathology, Juntendo Tokyo Koto Geriatric Medical Center, Tokyo, ${ }^{3}$ Department of Radiology, Juntendo Tokyo Koto Geriatric Medical Center, Tokyo, ${ }^{4}$ Department of Respiratory Medicine, Juntendo University, School of Medicine, Tokyo, ${ }^{5}$ Department of Molecular and Cellular Parasitology, Juntendo University, School of Medicine, Tokyo, ${ }^{6}$ Department of Pathology, Juntendo University, School of Medicine, Tokyo and ${ }^{7}$ Department of Parasitology, Asahikawa Medical College, Asahikawa

Received for publication December 24, 2007; Accepted for publication March 8, 2008

Correspondence to Dr. Mariko Yamazaki, mariko@med.juntendo.ac.jp 

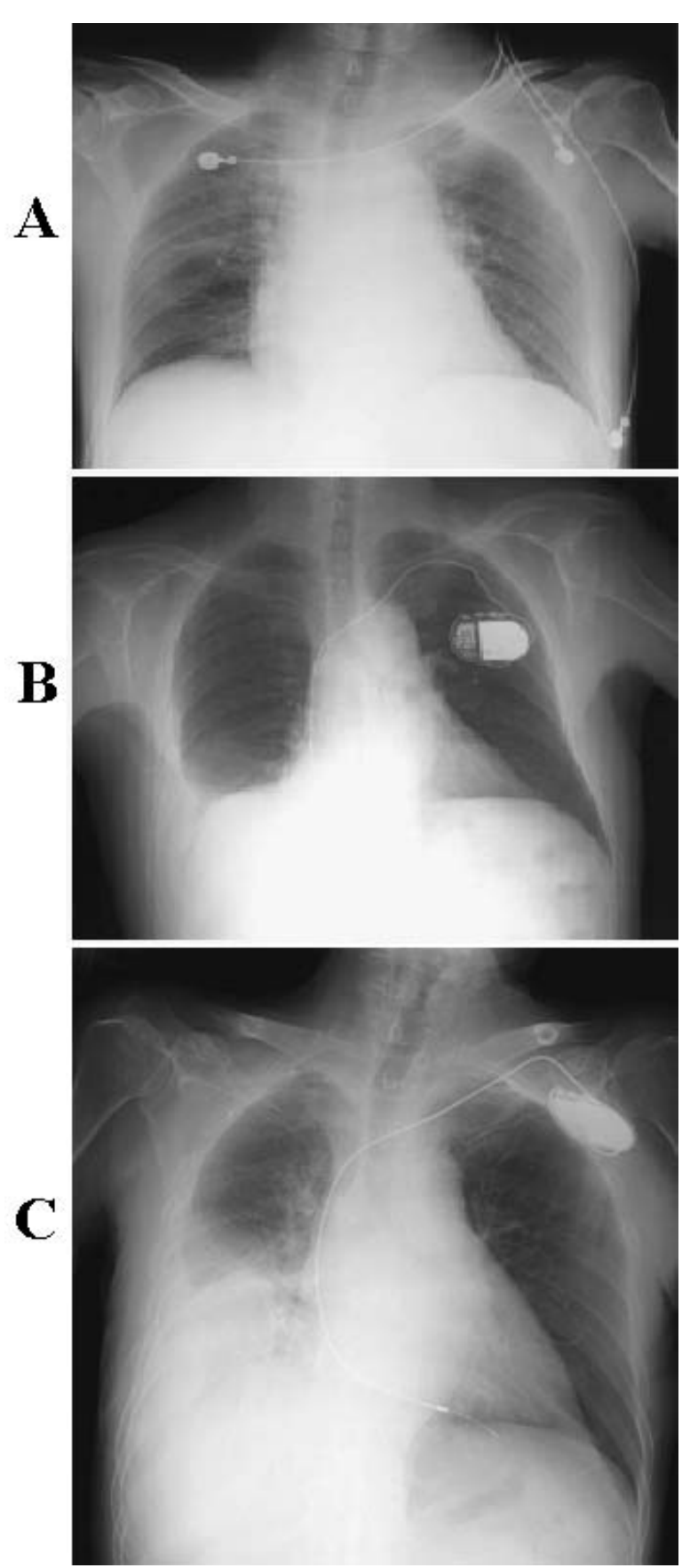

Figure 1. Chest radiographs. Chest radiograph at 16 months prior to admission reveals no sign of pleural effusion or pleural thickening (Panel A). Chest radiograph at $\mathbf{4 0}$ days earlier when the time of his admission at the former hospital revealing right pleural effusion and the location of an implanted permanent cardiac pacemaker (Panel B). Chest radiograph taken on admission at our hospital in the Fowler's posture position revealing the deterioration of the patient's right pleural effusion in comparison with his previous radiograph (Panel C).

characteristic eosinophilic pleural effusion (49\% of cells identified were eosinophils). Although no malignant cell or Mycobacterium tuberculosis (M. tuberculosis) was identified, the level of adenosine deaminase in the pleural effusion was slightly elevated at $40.7 \mathrm{IU} / \mathrm{L}$ (normal value, less than 40
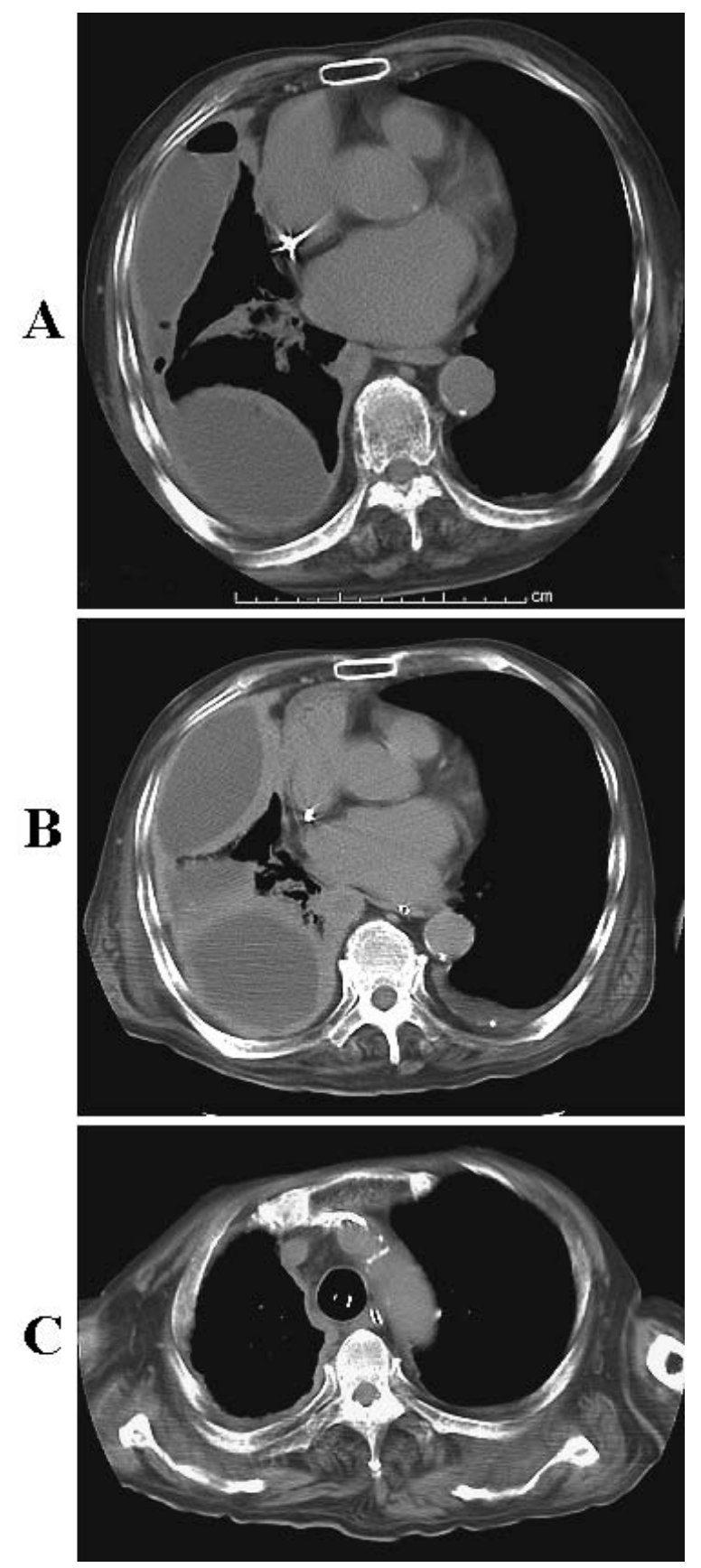

Figure 2. CT of the Thorax. Thoracic CT scan at admission identifying the air-liquid level and the right-sided pleural effusion encapsulated with thickened walls (Panel A). CT scan after the completion of treatment with Praziquantel revealed further deterioration of the plural effusion and an increase in the thickness of the encapsulating wall in comparison to his previous examination (Panel B). In addition, the thickening of the pleural wall extended to the level of right upper lung (Panel C).

IU/L). Systemic administration of prednisolone at the dosage of $15 \mathrm{mg}$ per day and anti-tuberculosis agents, isoniazid (200 mg per day) and rifampicin (300 mg per day) were begun on the suspicion of tuberculous pleuritis. After 40 days of hospitalization without any improvement of accumulation of pleural fluid, the patient was referred and transferred to our institution. Past medical history revealed left-sided hemi- 


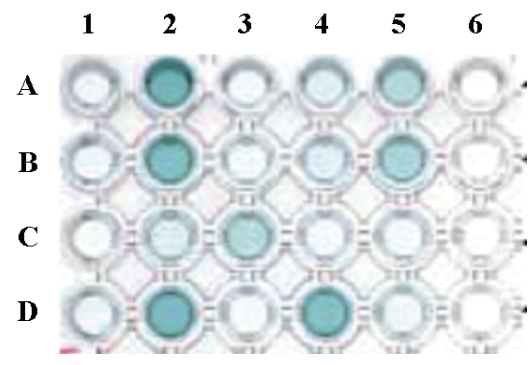

Figure 3. Enzyme-linked immunoabsorbent assay for various parasites. Lanes 1-6 indicate sera obtained from various patients with parasitic infection. Lane 1: serum obtained from negative control patient; lane 2: serum obtained from a patient with Paragonimus westermani infection; lane 3: serum obtained from a patient with Schistosoma mansoni infection; lane 4: serum obtained from a patient with Dirofilaria immitis infection; lane 5: serum obtained from the present case; and lane 6: blank. Rows A-D represent the antigens of various parasites; antigen of $P$. westermani (Row A), P. miyazakii (Row B), S. mansoni (Row C), D. immitis (Row D). The serum of the present case was positive for $P$. westermani (5-A) and $P$. miyazakii (5-B).

plegia resulting from right cerebral infraction secondary to atrial flutter, which had occurred 16 months earlier. Since then, dysphagia and excessive salivation were noted. Brain magnetic resonance imaging was performed in our hospital and an old infarction in the right middle cerebral arterial field was confirmed and there were no additional lesions identified. The patient underwent implantation of a cardiac pacemaker after experiencing multiple episodes of bradycardia due to atrial fibrillation a year ago. His work history revealed that he worked as a ship engineer for one year at age 21 and as a coal miner for 2 years until he finally settled down as an iron welder where he worked for 46 years until he retired at the age of 70 years. Chest radiographic examination performed 16 months earlier revealed no pleural thickening or effusion (Fig. 1A) but the radiographic examination conducted at the time of admission at his former hospital (40 days prior to referral to our hospital) revealed the existence of a right-sided pleural effusion (Fig. 1B). On admission to our hospital, deterioration of the right-sided pleural effusion was noted on the radiographic film (Fig. 1C). Thoracic CT scan revealed right-sided pleural fluid encapsulated with thick walls and an air-liquid level without parenchyma lesions (Fig. 2A). Aspirated pleural effusion revealed exudative straw-colored fluid, $\mathrm{pH}$ of 7.22 , lactate dehydrogenase of $1,440 \mathrm{IU} / \mathrm{L}$ (176 IU/L in serum), protein of 2.6 $\mathrm{mg} / \mathrm{dL}(5.6 \mathrm{mg} / \mathrm{dL}$ in serum), glucose of $42 \mathrm{mg} / \mathrm{dL}(79 \mathrm{mg} /$ $\mathrm{dL}$ in serum) and leukocyte count of 3,540 cells $/ \mathrm{mm}^{3}$. Leukocyte differential count was $56.5 \%$ lymphocytes, $21.8 \%$ eosinophils, $17.7 \%$ neutrophils and $4.0 \%$ monocytes (mononuclear cells). The level of hyaluronic acid in the pleural fluid was $0.00133 \mathrm{mg} / \mathrm{mL}$ (a cut-off level of $75 \mathrm{mg} / \mathrm{mL}$ was reported for malignant mesothelioma). Microbiological examination of the aspirated fluid specimen was negative for
Table 1. Peripheral Blood Eosinophil Count

\begin{tabular}{lc}
\hline $\begin{array}{l}\text { Time: before/after admission } \\
\text { at our hospital }\end{array}$ & $\begin{array}{l}\text { Eosinophils } \\
\left(\text { per } \mathrm{mm}^{3}\right)\end{array}$ \\
\hline 15 months before & 165 \\
12 months before & 224 \\
45 days before & 844 \\
25 days before & 1808 \\
on admission & 1755 \\
20 days after & 3312 \\
30 days after & 8202 \\
40 days after & 8278
\end{tabular}

M. tuberculosis, fungi and bacteria. Repeated cytological examinations of the aspirated fluid were negative for malignant cells. A chest tube was inserted into the right pleural space for the drainage of the pleural effusion when the diagnosis of empyema of unknown etiology was established. Urokinase was administered through the chest tube. On the third day of treatment, overt pneumothorax developed at the right thoracic space. Intra-thoracic administration of urokinase was terminated and the chest tube was kept in place for drainage. However, there was only slight improvement of the hydro-pneumothorax, and the chest tube was removed after 20 days. Then the pleural fluid filled the air space in the thoracic cavity and the pneumothorax was resolved. Tapering of the dosage of prednisolone and the discontinuation of anti-tuberculosis agents were conducted at the time of chest tube placement, and peripheral eosinophilia (defined as eosinophil count of $>400$ cell $/ \mathrm{mm}^{3}$ ) became evident (Table 1). The presence of hematological malignancy was omitted from the findings of bone marrow aspiration. Pleural biopsy using a Cope needle revealed the nonspecific inflammation, because the biopsied specimen contained only fibrous proliferation and degenerative mesothelial cells. The serum was examined for the presence of specific IgG antibodies against Paragonimus westermani, Paragonimus miyazakii, Schistosoma mansoni and Dirofilaria immitis with enzyme-linked immunosorbant assay (ELISA). The results of ELISA were positive for both Paragonimus westermani and Paragonimus miyazakii (Fig. 3). Ova for Paragonimus were not identified in sputum, pleural fluid or in the stool. The patient recalled eating fried fresh water crabs about 10 years ago but denied recent ingestion of raw or improperly cooked freshwater crab and crayfish or other foods known to harbor the parasite such as wild boar meat. There was no peripheral blood eosinophilia detected 12 months earlier when he underwent surgery for permanent cardiac pacemaker implantation (Table 1). From the positive results of ELISA, we repeatedly administered Praziquantel, orphan drug for treatment of Paragonimus with two treatments of 3day administration at a dose of $3,600 \mathrm{mg}(80 \mathrm{mg} / \mathrm{kg})$ per day with an interval of two weeks between treatments. However, there was no improvement in the condition of the pleural fluid. Thoracic CT scan revealed deterioration in pulmonary effusion and thickening of the encasing wall 

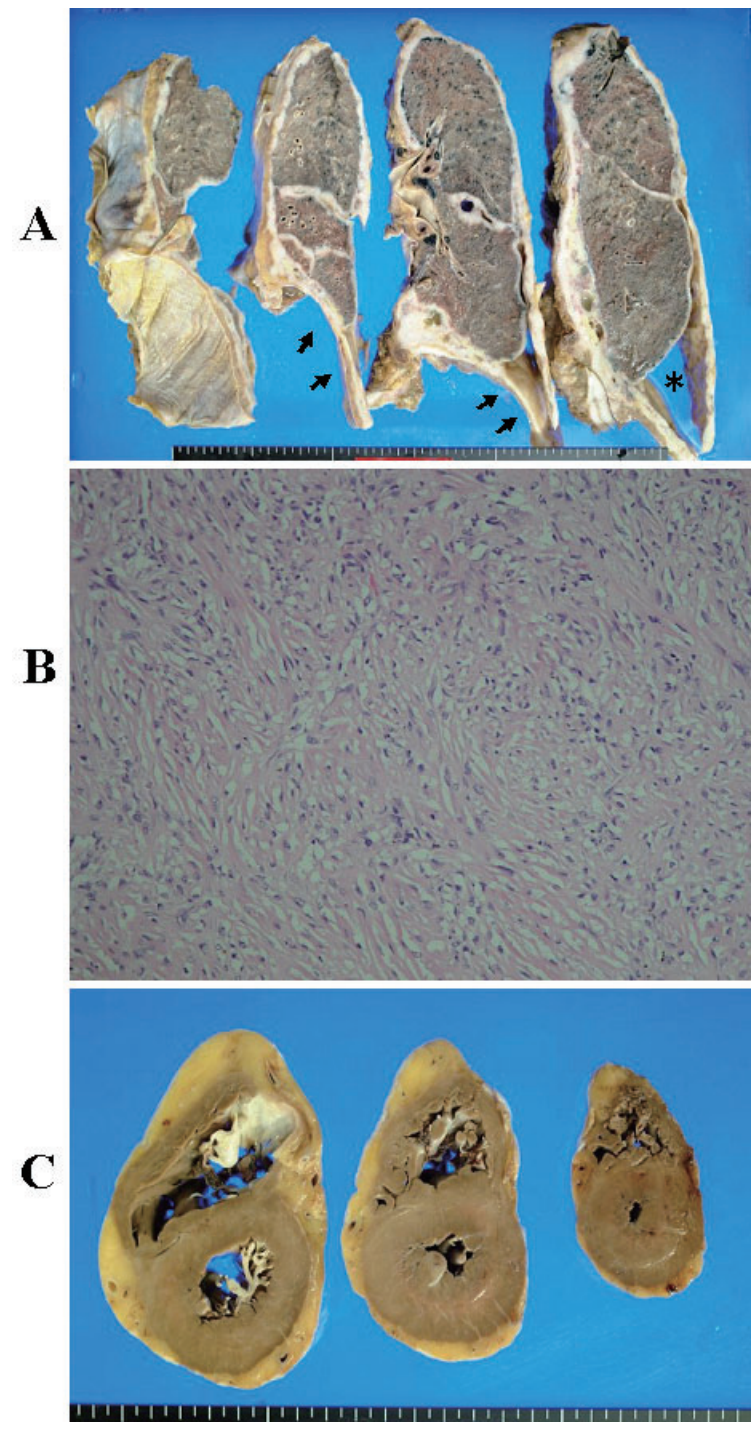

Figure 4. Pathological findings. The gross appearance of right whole lung is encased with thick white-colored fibrous tissue (Panel A). Cavitary lesion is identified in the posterolateral region of the right pleural space (asterisk) (Panel A). Note the right diaphragm was also involved and was diffusely thick (arrows). Microscopic appearance of the tissue encasing the right lung consisted of fibrous sarcomatous tissue stained with Hematoxylin and Eosin staining (Panel B). The gross appearance of heart revealed that the heart was encased with thick fibrous tissue of the malignant mesothelioma, which invaded into the myocardium (Panel $\mathrm{C}$ ).

(Fig. 2B). Rather the thickness of pleura extended to the level of the superior plane of the lung (Fig. 2C). There were no changes noted for the peripheral eosinophilia at one month (30 days after admission: Table 1). His condition gradually deteriorated until his death on the sixty-seventh day of hospitalization due to progressive congestive heart failure. Postmortem examination was performed. The gross appearance of the right thoracic cavity revealed that the whole right lung was covered with white-colored thick fibrous tissue (Fig. 4A). Hematoxylin and Eosin (HE) staining of the tissue demonstrated the proliferation of spindleshaped fibrous tumor cells (Fig. 4B). These tumor cells were negative for carcinoembryonic antigen (CEA), epithelioid mesothelioma markers calretinin and mesothelin. However, the tumor cells were positive for vimentin and cytokeratin. The intermediate filament cytoskeleton was also detected with electron microscopy. Together with HE staining and these findings, the pathological diagnosis of sarcomatous type of malignant mesothelioma was made. The heart was encased in a thick layer of malignant mesothelioma with illdefined margins indicating its invasiveness into myocardium (Fig. 4C). Old and recent onset myocardial necrosis, degenerative changes and scar formation of the heart were also identified. Cardiac involvement by malignant mesothelioma which restricted cardiac motion, together with repeated episodes of ischemic heart disease, were postulated to have resulted in fatal circulatory insufficiency. Pathological characteristics of pulmonary paragonimiasis, such as granuloma formation with or without ova of flukes were not identified in the lungs or pleura.

\section{Discussion}

The clinical feature of this patient to be discussed first is the condition of eosinophilia of the pleural fluid. Pleural effusion with greater than $10 \%$ eosinophils is often observed in cases with the presence of air or blood in the pleural space, such conditions are encountered after repeated thoracentesis, pneumothorax and traumatic hydrothorax (5). Asbestos-related effusion, pleural effusion secondary to drug reaction or parasitic diseases such as paragonimiasis, malignancy, and tuberculosis are also listed as causes (6). We considered that the prior episode of pneumothorax was rather masked with effusion than de novo pneumothorax and drainage with the chest tube clarified the presence of the pneumothorax. We first speculated that air exposure of the pleural space was a causative factor to produce pleural eosinophilia. However, with tapering the dosage of systemically administered steroid, peripheral blood eosinophilia (20 days after admission: Table 1) became evident. Parasitic infection and hematological malignancy are considered to respond to steroid administration. Hematological malignancy was ruled out from the findings of bone marrow aspiration. Spontaneous eosinophilia in peripheral blood associated with mesothelioma is extremely rare (7). Parasitic infection is listed as the top candidate for eosinophilia of pleural effusion and peripheral blood.

The issue to discuss secondly is whether or not pulmonary paragonimiasis was present in this case. Thoracic CT findings of pulmonary paragonimiasis include pulmonary effusion, hydropneumothorax, pulmonary nodules, consolidation, and cysts $(8,9)$. Pleural involvement without the presence of parenchymal lung lesion as in the present case is often encountered among the known indicated findings (8, 9). There were no parasitic ova identified in the stool, sputum and/or pleural effusion in our case. However, immunoblot analysis for Paragonimus specific antibodies is presently the most reliable tool for diagnosis with a sensitiv- 
ity of $90 \%$ and a specificity of $99 \%$ (10). Since ELISA analyses were positive for the presence of antibodies specific to Paragonimus, we diagnosed the present case as having pulmonary paragonimiasis with eosinophilic pleural effusion and peripheral eosinophilia.

The latent period between the time of ingestion of contaminated foods and time of appearance of overt symptoms is speculated to be two to three months, although there are much controversies and divergence among clinical reports such as 4, 7, 18 months (11-15). The present patient denied recent ingestion of raw or improperly cooked freshwater crabs, crayfishes, and wild boar meats. Jeon et al reported in their recent article that approximately $30 \%$ of the patients in Korea with pulmonary paragonimiasis denied history of eating paratenic hosts (15). As for the treatment of pulmonary paragonimiasis, it is recommended to administer praziquantel at the dose of $75 \mathrm{mg}$ per kilogram for 2 or 3 days. Mukae et al reported that the responsiveness of patients with pulmonary paragonimiasis to praziquantel at this recommended dose is excellent (2) and that all thirteen Japanese patients recovered from pleural effusion. Clinical response in the present case was poor even after two treatments of praziquantel and the final outcome was death due to cardiovascular causes. Cases of paragonimiasis leading to fatal outcomes were observed in patients with cerebral involve- ment such as cerebral vascular accident and other hemorrhagic consequences (3). Since cerebral involvement was not evident in our case, the clinical course of our patient was considered as a bizarre case for pulmonary paragonimiasis.

Postmortem examination revealed malignant mesothelioma encasing the right pleural space and invading the heart. He had a high risk of malignant mesothelioma with a positive occupational history. Chest radiographic examination taken 16 months earlier was unremarkable; the presence of pleural thickening or effusion was not confirmed, which is often observed in malignant mesothelioma. Since postmortem examination did not reveal any characteristic pathological findings of paragonimiasis such as ova or granulated tissue, we could not speculate whether the infection was fresh or old. However, peripheral blood eosinophilia was evident 45 days before admission (refer to Table 1), suggesting recent infection with paragonimus flukes. This is a very rare case of pulmonary paragonimiasis with coincidental malignant mesothelioma.

\section{Acknowledgement}

The authors would like to thank Dr. Tomonobu Okuno of Shin-Katsushika Hospital and Dr. Hitoshi Saitoh of Heisei Tateishi Hospital for their assistance and contribution of the chest radiographs.

\section{References}

1. Obara A, Nakamura-Uchiyama F, Hiramatsu K, Nawa Y. Paragonimiasis case recently found among immigrants in Japan. Internal Med 43: 388-392, 2004.

2. Mukae H, Taniguchi H, Matsumoto N, et al. Clinicoradiologic features of pleuropulmonary Paragonimus westermani on Kyusyu Island, Japan. Chest 120: 514-520, 2001.

3. Choo JD, Suh BS, Lee HS, et al. Chronic cerebral paragonimiasis combined with aneurysmal subarachnoid hemorrhage. Am J Trop Med Hyg 69: 466-469, 2003.

4. Dainichi T, Nakahara T, Moroi Y, et al. A case of cutaneous paragonimiasis with pleural effusion. Int J Dermatol 42: 699-702, 2003.

5. Ligh RW. Pleural Diseases. 4th ed. Lippincott Williams \& Wilkins, Philadelphia, 2001: 48-50.

6. Martinez-Garcia MA, Cases-Viedma E, Cordero-Rodriguez PJ, et al. Diagnostic utility of eosinophils in the pleural fluid. Eur Respir J 15: 166-169, 2000.

7. Davis BH. Hypereosinophilia associated with a peritoneal mesothelioma. Arch Pathol Lab Med 103: 487, 1979.

8. Im JG, Wang HY, Kim WS, Han MC, Shim YS, Chow SY. Pleuropulmonary paragonimiasis: radiologic findings in 71 patients. AJR Am J Roentgenol 159: 39-43, 1992.
9. Kim TS, Han J, Shim SS, et al. Pleuropulmonary paragonimiasis: CT findings in 31 patients. AJR Am J Roentgenol 185: 616-621, 2005.

10. Robertson KB, Janssen WJ, Saint S, Weinberger SE. Clinical problem-solving. The missing piece. N Engl J Med 355: 19131918, 2006.

11. Uchida K, Sekiguchi S, Doi Y, Yamazaki H. Pulmonary paragonimiasis with pleural effusion containing paragonimus ova: sonographical appearance of pleural effusion. Intern Med 34: 1178-1180, 1995.

12. Inoue $\mathrm{Y}$, Kawaguchi $\mathrm{T}$, Yoshida A, et al. Paragonimiasis miyazakii associated with bilateral pseudochylothorax. Intern Med 39: 579582, 2000.

13. Nakamura-Uchiyama F, Onah DN, Nawa Y. Clinical features of paragonimiasis cases recently found in Japan: parasite-specific immunoglobulin $\mathrm{M}$ and $\mathrm{G}$ antibody classes. Clin Infect Dis 32: e 151-e153, 2001.

14. DeFrain M, Hooker R. North American paragonimiasis: case report of a severe clinical infection. Chest 121: 1368-1372, 2002.

15. Jeon K, Koh WJ, Kim H, et al. Clinical features of recently diagnosed pulmonary paragonimiasis in Korea. Chest 128: 1423-1430, 2005 .

(C) 2008 The Japanese Society of Internal Medicine

http://www.naika.or.jp/imindex.html 